\title{
Very Low and High Blood Viscosity are Risk Factors for Internal Flow Choking Causing Asymptomatic Cardiovascular Disease
}

\section{V.R. Sanal Kumar ( $\nabla$ vr_sanalkumar@yahoo.co.in ) \\ Indian Space Research Organisation, VSSC}

Shiv Kumar Choudhary

All India Institute of Medical Sciences

Pradeep Kumar Radhakrishnan

GITAM University

R. S. Bharath

Indian Institute of Science

Nichith Chandrasekaran

Indian Institute of Science

Vigneshwaran Sankar

Indian Institute of Science

Ajith Sukumaran

Kumaraguru College of Technology

Charlie Oommen

Indian Institute of Science

\section{Research Article}

Keywords: Acute-Heart Failure, Asymptomatic hemorrhage, Biofluid choking, Covid-19, BHCR, Sanal flow choking.

Posted Date: September 16th, 2021

DOI: https://doi.org/10.21203/rs.3.rs-151850/v2

License: (c) (1) This work is licensed under a Creative Commons Attribution 4.0 International License.

Read Full License 


\section{Very Low and High Blood Viscosity Are Risk Factors for}

2 Internal Flow Choking Causing Asymptomatic

\section{Cardiovascular Disease}

4 Short Title: Low BP ratio and/or high BHCR reduce the risk of Covid-19

5 V. R. Sanal Kumar, Ph.D, PDF ${ }^{1,25^{*}}$, Shiv Kumar Choudhary, M.S., M.Ch, FNAMS ${ }^{3}$, Pradeep

6 Kumar Radhakrishnan, M.S, M.Ch, CTVS (AIIMS), PDF ${ }^{3,4}$, R.S.Bharath M.Sc ${ }^{2}$, Nichith

7 Chandrasekaran, M.S ${ }^{2,5}$, Vigneshwaran Sankar, M.Tech, ${ }^{2,5}$ Ajith Sukumaran,M.S, ${ }^{5}$ and

8 Charlie Oommen, Ph.D ${ }^{2}$

$9 \quad{ }^{1}$ Indian Space Research Organisation, VSSC, Trivandrum 695 022, Kerala, India

$10 \quad{ }^{2}$ Indian Institute of Science, Bangalore, Karnataka, 560012, India

$11{ }^{3}$ All India Institute of Medical Sciences, New Delhi - 110608, India

$12 \quad{ }^{4}$ GITAM University, Visakhapatnam-530045, Andhra Pradesh, India

$13{ }^{5}$ Kumaraguru College of Technology, Coimbatore - 641 049, Tamil Nadu, India

$14 *$ Corresponding author name: V.R.Sanal Kumar

15 Email: vr_sanalkumar@yahoo.co.in

16 Disclosure Statement of Authors: Nothing to disclose

17 Conflicts of interest: None

18 Competing financial interests: None

19 Word Count: 6031 (Body text and Figure legends)

20 Keywords: Acute-Heart Failure, Asymptomatic hemorrhage, Biofluid choking, Covid-19,

21 BHCR, Sanal flow choking. 


\section{Abstract}

\section{Background}

24 It is well known that the common aftereffect of administration with the blood-thinning-drug for

25 lowering the blood-viscosity is bleeding. And very often during the blood-thinning medication,

26 without any preceding symptoms, asymptomatic-hemorrhage ( $\mathrm{AH})$ and the acute-heart-failure

27 (AHF) are testified. Of late (2021) researchers reported (PMID: 34326352) that symptoms are

28 intensifying on asymptomatic cardiovascular-diseases and the various types of neurological-

29 disorders linked with COVID-19 pandemic due to the nanoscale Sanal-flow-choking.

\section{Objectives}

31 Our aim was to establish the proof of the concept of the Sanal flow choking in cardiovascular

32 system (CVS) causing AH and AHF by correlating multitude of parameters, viz., the blood 33 pressure ratio (BPR), biofluid/blood-heat-capacity-ratio (BHCR), blood viscosity, blood 34 density, stenosis (in terms of the vessel cross-sectional area and/or the hydraulic diameter of the 35 vessel) and ejection fraction in terms of fluid flow rate. In this regard an infallible closed-form 36 analytical model was aimed to develop. We also aimed for correlating multitude of variables for

37 setting unchoked flow conditions in CVS for negating AH and AHF. We were scheduled 38 in vitro and in silico studies for corroborating the proof of the concept of flow choking in CVS.

\section{Methods}

40 The proof of the concept of Sanal-flow-choking and unchoked flow conditions in CVS are 41 established using the closed-form-analytical-methodology by setting with the multitude of 42 variables. Speciation analyses of blood samples of healthy subjects (human being/Guinea pig) 43 are carried out for the BHCR estimation of blood samples of healthy subjects (human 44 being/Guinea pig) by invoking in vitro method. Asymptomatic pressure-overshoot due to the 
45 Sanal flow choking and transient shock wave generation in a simulated artery (a case with gas

46 embolism) with a divergence region is demonstrated using in silico method.

\section{$47 \quad$ Results}

48 We established the proof of the concept of internal flow choking in CVS causing 49 cardiovascular risk through the closed-form analytical, in vitro and in silico methods. We 50 discovered that at a critical blood-pressure-ratio (BPR), the internal-flow-choking occurs 51 anywhere in the CVS with the sudden expansion/ divergence/ bifurcation / stenosis / occlusion

52 or vasospasm regions without prejudice to the percutaneous coronary intervention (PCI).

53 Mathematical models disclose that the reasonably high and low blood viscosity are risk factors

54 of asymptomatic cardiovascular disease. In vitro results reveal that nitrogen $\left(\mathrm{N}_{2}\right)$, oxygen $\left(\mathrm{O}_{2}\right)$,

55 and carbon dioxide $\left(\mathrm{CO}_{2}\right)$ are the leading gases evolved from fresh-blood samples of the healthy

56 subjects (human-being and Guinea-pig) at a temperature range of $37-40^{\circ} \mathrm{C}\left(98.6-104^{0} \mathrm{~F}\right)$. It

57 increases the risk of flow-choking leading to asymptomatic cardiovascular-diseases and the

58 various types of neurological-disorders. This physical situation is more critical for the COVID-

5919 patients as it leads to AH and AHF. We observed through in vitro studies that the healthy

60 Guinea-pig is having thermal-tolerance level higher than the human being in terms of BHCR.

61 The possible episode of Sanal flow choking and shock wave generation in an artery with the

62 divergent/ bifurcation region is demonstrated through in silico methods.

\section{Conclusions}

64 An over dose of blood-thinning drug for decreasing the viscosity will enhance the Reynolds 65 number, which creates high turbulence level causing an augmented boundary layer blockage

66 factor leading to an early undesirable biofluid/Sanal flow choking. It enhances the possibilities

67 of cavitation and the Sanal-flow-choking heading to the shock wave generation followed by 
68 transient pressure-overshoot causing memory effect (stroke history) in viscoelastic vessels.

69 Determining the exact blood-thinning dose is critical for achieving the preferred therapeutic

70 efficacy and annulling undesirable flow-choking causing asymptomatic-hemorrhage (AH) and

71 the acute-heart-failure (AHF). We could establish herein that the lopsided blood-thinning

72 administration enhances the risk of the Sanal-flow-choking due to the augmented boundary layer

73 blockage (BLB) factor. We concluded that cardiovascular risk could be reduced by

74 simultaneously lessening the blood-viscosity and flow turbulence by increasing thermal-

75 tolerance-level in terms of BHCR and/or by decreasing the blood pressure (BP) ratio.

\section{Condensed Abstract}

77 Herein, we established the proof of the concept of internal flow choking in CVS causing 78 cardiovascular risk through the closed-form analytical, in vitro and in silico methods. An over 79 dose of blood-thinning drug will enhance the Reynolds number, which creates high turbulence 80 level causing an augmented boundary layer blockage factor leading to an early undesirable 81 biofluid/Sanal flow choking at a critical blood-pressure-ratio (BPR). The fact is that in nanoscale 82 vessels when the pressure of fluid increases, average-mean-free-path decreases and thus, the 83 Knudsen number reduces. It leads to the physical situation of no-slip boundary condition with 84 compressible-viscous flow effect. Sanal-flow-choking is a compressible-viscous flow effect 85 establishing a physical condition of the sonic-fluid-throat, at a critical blood pressure ratio 86 (BPR). We concluded that asymptomatic-hemorrhage (AH) and acute-heart-failure (AHF) are

87 transient-events as a result of internal flow-choking in nanoscale and/or large vessels followed 88 by the shock wave creation and transient pressure-overshoot. We concluded that cardiovascular 89 risk could be reduced by simultaneously lessening the blood-viscosity and flow turbulence by 
90 increasing thermal-tolerance-level in terms of BHCR and/or by decreasing the blood pressure

91 (BP) ratio.

\section{Introduction}

93 The theoretical discovery of the nanoscale Sanal-flow-choking [1] is a paradigm shift in risk 94 assessment for hemorrhagic stroke and other neurological disorders. This study is the 95 continuation of our previous work published in Nature Scientific Reports [1]. Of late (2021) 96 researchers reported that data are increasing on the various asymptomatic cardiovascular

97 diseases associated with COVID-19 pandemic [1-3] due to the Sanal flow choking [1, 4-6] 98 (PMC7267099). Through our companion papers we have reported that Sanal flow choking leads 99 to asymptomatic aneurysm [6], hemorrhagic-stroke [7], acute myocardial infarction [8] and 100 other neurological-disorders [4-14] on Earth and Human spaceflight if the vessel geometry is having 101 divergence, bifurcation, stenosis and/or occlusion regions. In this paper we are correlating 102 multitudes of variables causing internal flow choking leading to AH and AHF. There are two 103 types of internal flow choking (see Fig. 1(a-c)), viz., (i) biofluid/blood flow choking due to 104 plaque or stenosis or occlusion (i.e., geometry effect of vessels), (ii) Sanal flow choking due to 105 boundary layer blockage (i.e., fluid dynamics effect) [11].

106 The acute-heart-failure (AHF) is known as the fatal disease worldwide over the centuries. 107 Very often, the fatal AHF occurs without prior indications of coronary artery obstruction 108 (angina). According to the WHO [15], the Ischemic heart disease (IHD) [16] and asymptomatic 109 hemorrhage $(A H)$ / stroke are the world's biggest killers. M.Packer [17] categorically reported 110 that the acute-heart-failure (AHF) is a transient event and not a disease and put forward a 111 coherent claim for multidisciplinary research for drugs-discovery [18]. V.R.S.Kumar et al. [1, 112 4-14] reported in a series of connected papers that such transient events instigating the 


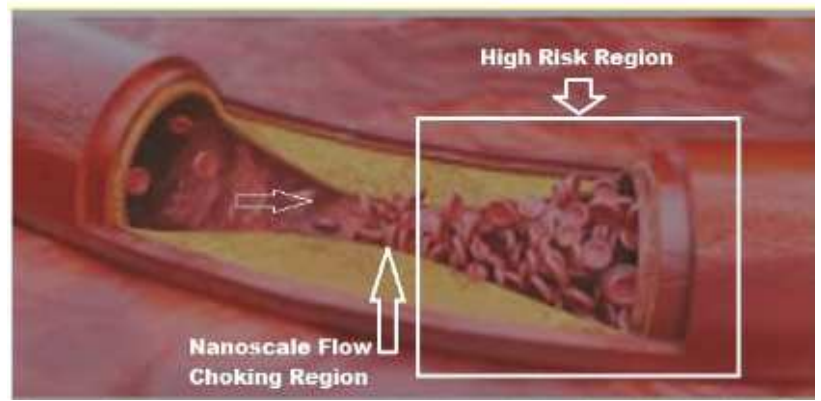

(a) Nanoscale flow choking in an artery with the plaque.

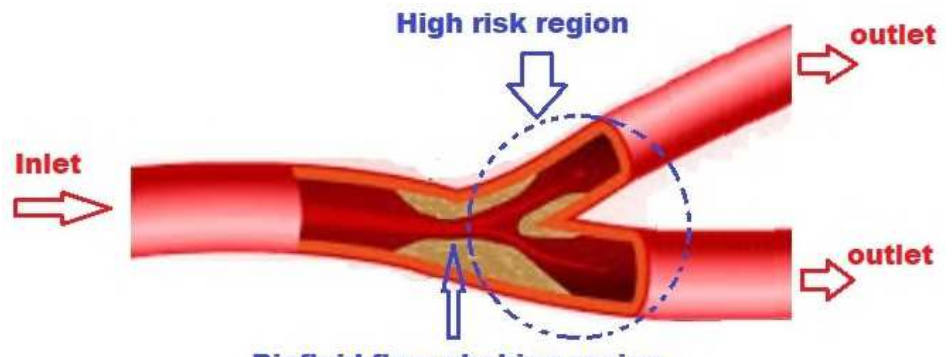

(b) Biofluid/blood flow choking in an artery with plaque and bifurcation.

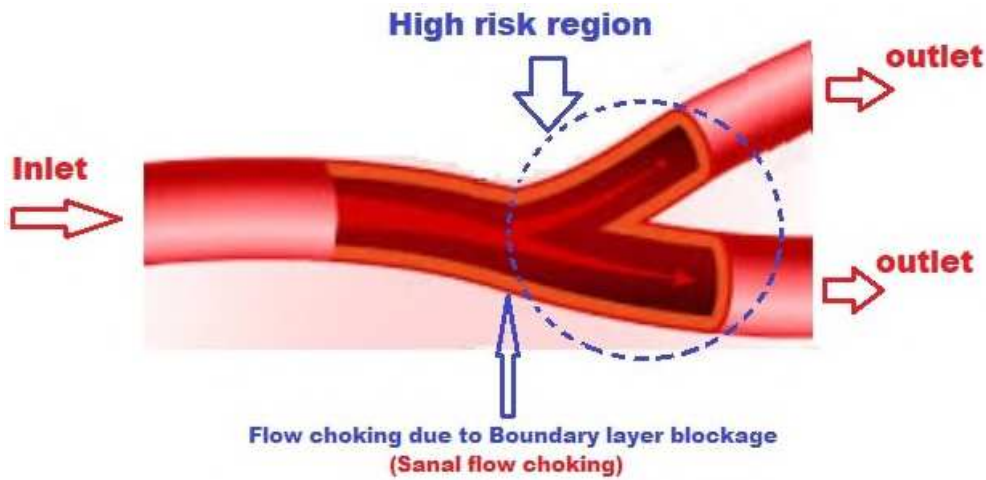

(c) Sanal flow choking in an artery with bifurcation and without plaque 


\section{Internal Flow Choking: The Central Illustration}

126

127

128

129

130

131

132

133

134

135

136

137

138

139

140

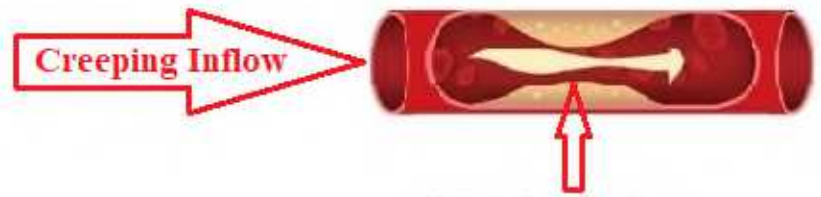

Biofluid Choking

(a)

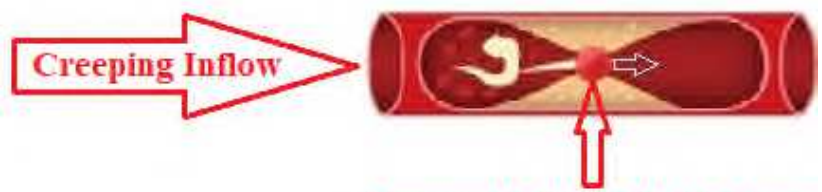

Nanoscale Biofluid Choking

(b)

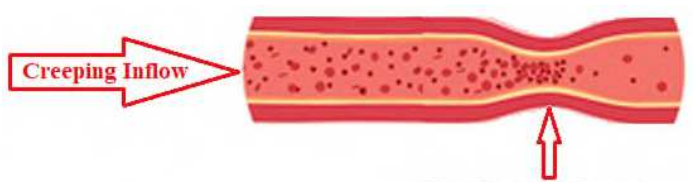

Biofluid Choking

(c)

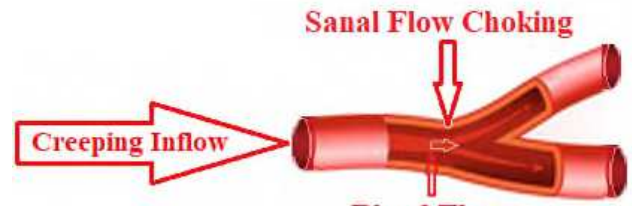

(d)

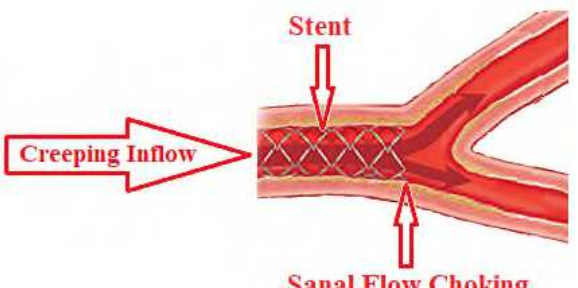

(e)

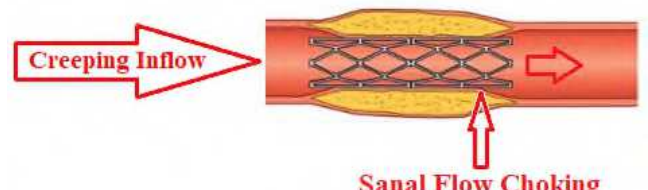

(f) 


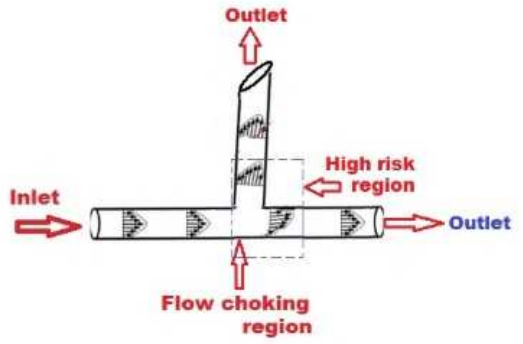

(g)

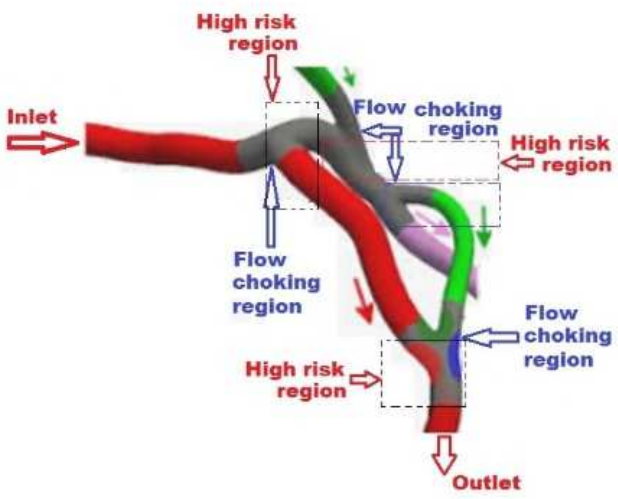

(i)
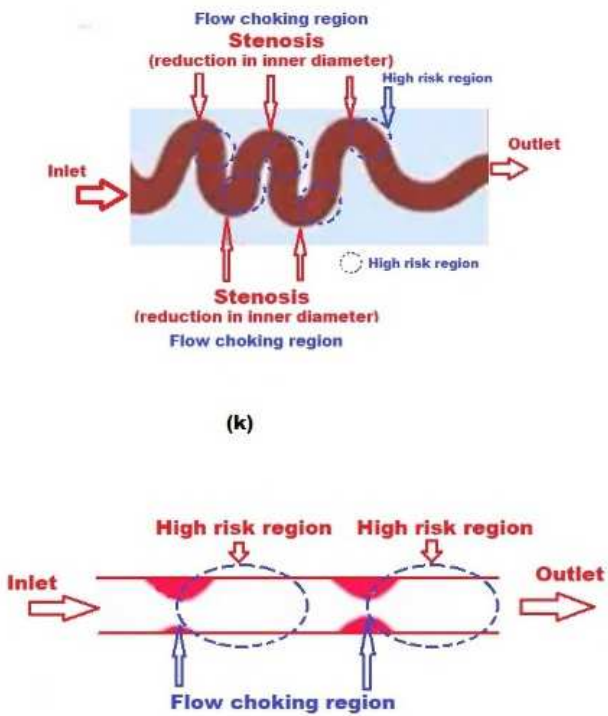

(m)

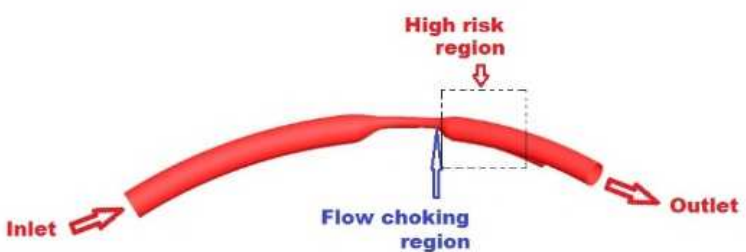

(h)

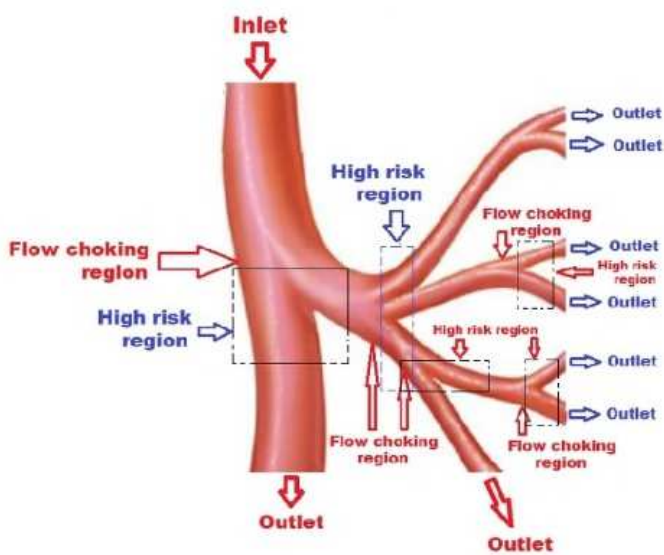

(j)

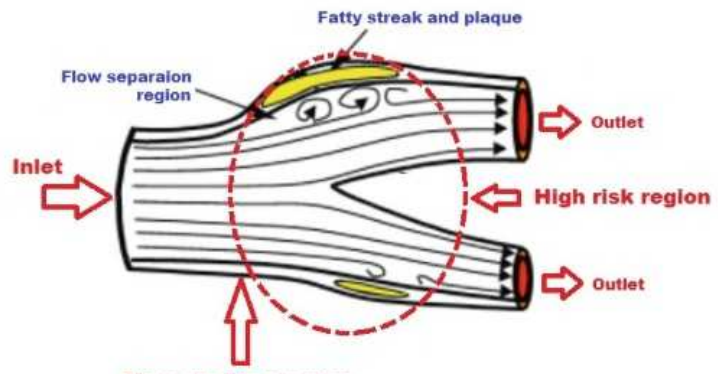

Flow choking region

(I)

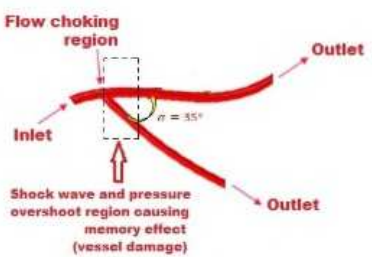

(n)

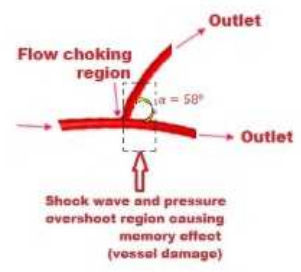

(o)

142 Figure 2(a-o). The demonstration of various physical situations of Internal flow choking

143 (Biofluid/Sanal flow choking) in the cardiovascular system without prejudice to 144 Percutaneous Coronary Intervention (PCI). 
145 cardiovascular diseases are due to an internal flow choking (biofluid and/or Sanal flow choking 146 - see Fig.1(a-c) and Fig.2(a-o)). It is followed by shock wave generation and pressure-

147 overshoot in the cardiovascular system (CVS), which happens at a critical systolic-to-diastolic 148 blood-pressure-ratio (BPR).

149 Internal flow choking could occur without prejudice to the Percutaneous Coronary 150 Intervention (PCI). The real scientific truth is that, at a critical BPR, the internal flow choking 151 followed by shock wave generation occurs anywhere in the CVS with sudden 152 expansion/divergence, vasospasm, bifurcation, stenosis and/or occlusion regions (see Fig.2(a-o) 153 - the central illustration). The critical fact is that, the internal biofluid/blood flow choking is 154 uniquely regulated by the heat-capacity-ratio (HCR) of the fluid (blood / biofluid / gas). It has 155 been established that the Sanal flow choking phenomenon occurs, in the real world flows 156 (continuum and non-continuum) due to the compressible viscous flow effect, in the form of a 157 sonic-fluid-throat effect as a result of BLB factor [1, 4-14]. Note that altered variations of blood 158 viscosity and turbulence lead to flow choking in CVS. It leads to cavitation, shock wave 159 generation and transient pressure-spike. Internal flow choking (biofluid / Sanal flow choking) 160 could happen in all vessels including vasa vasorum and nanoscale tubes [1]. The fact is that in 161 nanoscale vessels when the pressure of fluid increases, average-mean-free-path decreases and 162 thus, the Knudsen number reduces. It leads to the physical situation of no-slip boundary 163 condition with compressible-viscous flow effect [1]. Sanal-flow-choking is a compressible164 viscous flow effect establishing a physical condition of the sonic-fluid-throat, at a critical blood 165 pressure ratio $(\mathrm{BPR}=\mathrm{SBP} / \mathrm{DBP})$ due to the BLB factor. The concepts of Sanal flow choking $166[1,4-6]$ is well connected herein with the prevailing concepts in the biological sciences for 
167 discovering possible methods for negating the risk of biofluid/Sanal flow choking heading to 168 asymptomatic cardiovascular diseases.

169 Analytical Methodology

170 Using the compressible flow theory, the following closed-form analytical models (see Eqs.1-

171 5) have been developed for correlating the BHCR, BPR, biofluid/blood-kinematic-viscosity,

172 biofluid/blood-density, diastolic-blood-pressure (DBP), hydraulic-diameter of the vessel, the 173 vessel cross-sectional area, blood/biofluid velocity, Reynolds number $(R e)$, boundary-layer174 blockage (BLB) and ejection-fraction in terms of biofluid/blood flow rate (BFR) for predicting 175 the risk of flow-choking in cardiovascular system (CVS) causing asymptomatic-hemorrhage $176(\mathrm{AH})$ and the acute-heart-failure (AHF).

177 Equations $1 \& 2$ are two independent and complementing conditions for maintaining the 178 unchoked flow condition in the CVS. Note that flow gets choked when the flow Mach number 179 (M) reaches one. Therefore, it is mandatory to retain the flow Mach number always less than 180 one for prohibiting the internal flow choking in CVS, which is reflected in Eq.2, Eq.2a and 181 Eq.2b with the multitude of variables. Note that Eq.2a and Eq.2b are the corollary of Eq.2, which 182 explain the role of the vessel blockage, in terms of the vessel cross-sectional area and the 183 ejection fraction in terms of biofluid/blood flow rate (BFR), on the risk of flow choking leading 184 to $\mathrm{AH}$ and $\mathrm{AHF}$.

$$
B P R=\frac{S B P}{D B P}<\left(\frac{B H C R+1}{2}\right)^{B H C R / B H C R-1}
$$




$$
\frac{(\text { Reynold number })(\text { kinematic viscosity of blood/biofluid })}{\text { Hydraulic diameter of the vessel }}\left[\frac{\text { Blood density }}{(B H C R)(D B P)}\right]^{1 / 2}<1
$$

$$
\left[\frac{(\text { Blood / biofluid flow rate })_{\text {local }}(\text { blood/biofluid viscosity })_{\text {local }}}{(\text { BHCR })_{\text {lowest }}(\mathrm{DBP})(\text { Vessel cross }- \text { sectional area })_{\text {local }}}\right]^{1 / 2}<1
$$

$$
L C H I=\left(\frac{(B H C R)_{\text {lowest }}+1}{2}\right)^{B H C R_{\text {lowest }} / \text { BHCR }_{\text {lowest }}-1}
$$

$$
U C H I=\left(\frac{\left(\text { BHCR }_{\text {blood }}+1\right.}{2}\right)^{(\text {BHCR })_{\text {blood }} /\left(\text { BHCR }_{\text {blood }^{-1}}\right.}
$$

191 Note that for prohibiting the internal flow choking in CVS all subjects must maintain BPR lower 192 than the lower-critical-hemorrhage-index (LCHI), which could be estimated from the lowest 193 value of the BHCR of evolved gases in the CVS (see Eq.3). For instance, if carbon dioxide is 194 the dominant gas in the CVS it is mandatory to maintain BPR lower than 1.8257 for creating an 195 unchoked flow condition for prohibiting the shock wave generation and transient pressure196 overshoot causing the AH and AHF. The LCHI can be estimated through in vitro study aiming 197 for finding the dominant gases evolved from blood samples of each subject (human being or 198 animal) at different thermal levels. The upper critical hemorrhage index (UCHI) can be 199 predicted (see Eq.4) from the specific heat of blood at constant pressure $\left(\mathrm{C}_{\mathrm{p}}\right)$ and the specific 200 heat of blood at constant volume $\left(\mathrm{C}_{\mathrm{v}}\right)$, estimated using the Differential Scanning Calorimeter 201 Perkin Elmer DSC 8000. 
The boundary-layer-blockage (BLB) in the blood vessels can be predisposed by the variations in the biofluid viscosity and the BHCR of the flowing gas / nano plasma. Equation 5 relates the artery diameter $\left(\mathrm{d}_{\mathrm{i}}\right)$, the inflow Mach number $\left(\mathrm{M}_{\mathrm{inlet}}\right)$, the axial Mach number $\left(\mathrm{M}_{\mathrm{axial}}\right)$, and the BHCR, which is derived from compressible flow theory [4-6].

$$
B L B=\left[1-\left[\frac{M_{\text {inlet }}}{M_{\text {axis }}}\right]^{1 / 2}\left[\frac{1+\left(\frac{B H C R-1}{2}\right) M_{\text {axis }}^{2}}{1+\left(\frac{B H C R-1}{2}\right) M_{\text {inlet }}^{2}}\right]^{\frac{B H C R+1}{4(B H C R-1)}}\right] d_{i}
$$

208 The earlier researchers, all together, presumed that the human blood is an incompressible fluid 209 (i.e., $\mathrm{C}_{\mathrm{p}}=\mathrm{C}_{\mathrm{v}}$ ). Such an assumption is obviously not true as the human blood specific volume (or 210 density) does change with temperature or pressure. Note that all fluids in nature are compressible 211 [6-11] and therefore $C_{p}$ is always greater than $C_{v}[6]$. It establishes that the Sanal flow choking 212 and shock wave generation occurs at UCHI in CVS having divergent or bifurcation regions 213 without any plaque. At the Sanal flow choking condition, the creeping flow (low subsonic flow) 214 will get augmented in a uniform cross-sectional area duct due to the area blockage caused by 215 the boundary-layer-displacement-thickness (i.e., BLB in Eq.5). The total 3D boundary layer 216 blockage (TBLB) at Sanal-flow-choking condition $\left(\mathrm{M}_{\text {axis }}=1\right)$ for diabatic flows is obtained as 217 (see Eq.5a) [5-7],

$$
\left.T B L B\right|_{@ \text { sonic-fluid -throat }}=\left[1-M_{\text {inlet }} 1 / 2\left[\frac{2}{(B H C R)_{\text {lowest }}+1}\left(1+\frac{(B H C R)_{\text {lowest }}-1}{2} M_{\text {inlet }}^{2}\right)\right]^{\frac{(B H C R)_{\text {lowest }}+1}{4\left(1-(B H C R)_{\text {lowest }}\right)}}\right] d_{i}
$$




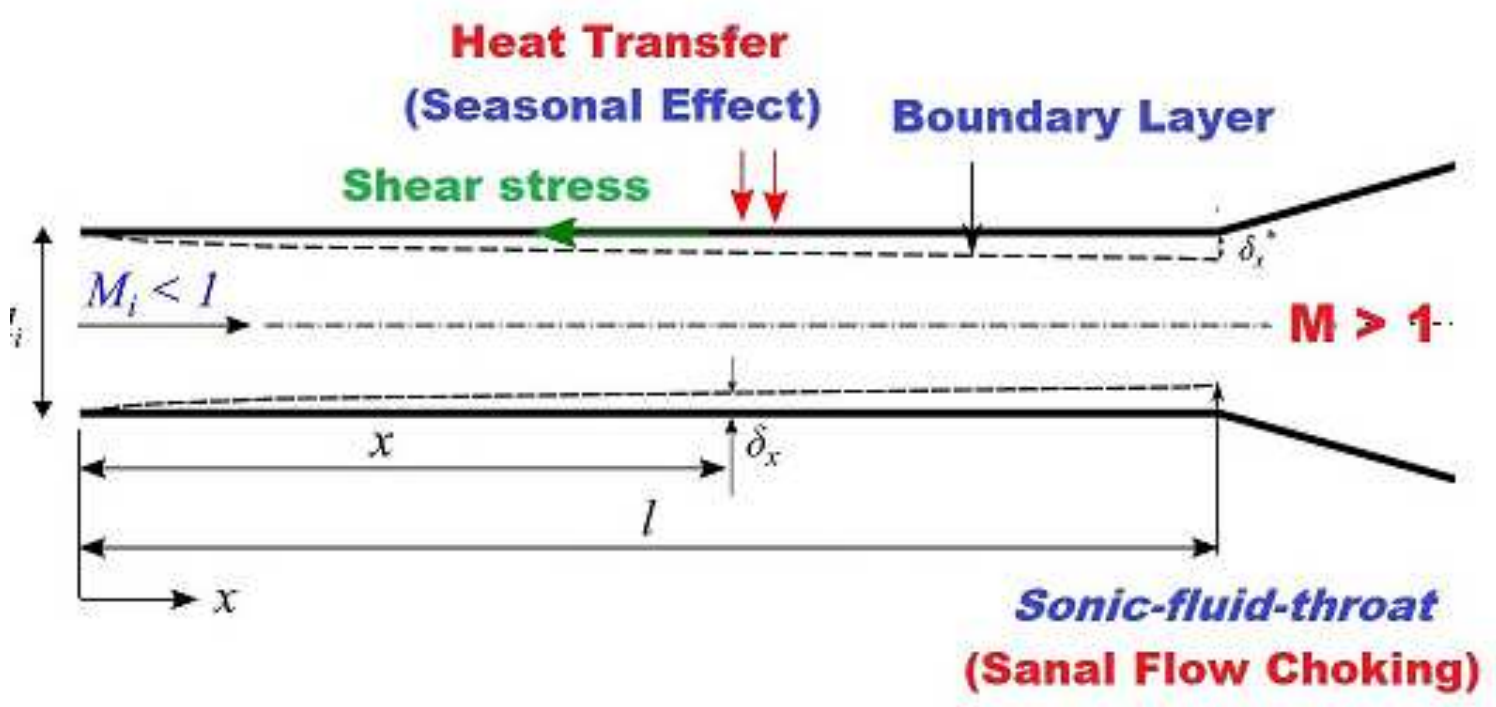

(a) An idealized physical model of an artery with divergent region

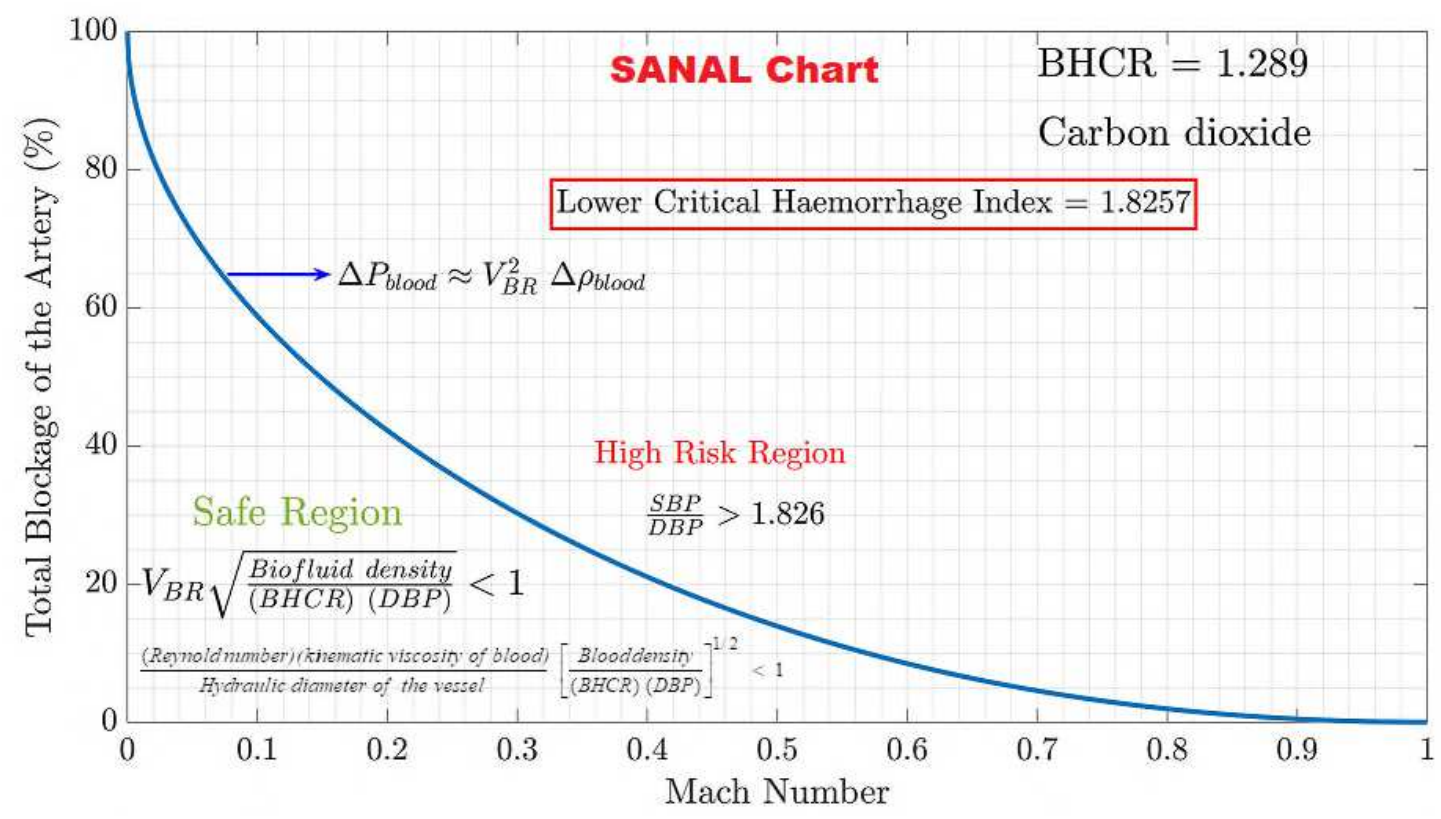


The BLB factor in the blood vessels could alter due to the seasonal effects (see Fig.3(a)) as

226 a result of the variations in the biofluid/blood viscosity [4-14, 19-24]. If the blood vessel

227 geometry is similar to the convergent-divergent (CD) nozzle shape (due to various physical

228 situations as seen in Fig. 1(a-c) \& Fig. 2(a-o)), the divergent region of the CD duct creates

229 supersonic flow immediately after the internal flow choking (biofluid / Sanal flow choking). It

230 leads to shock wave generation and transient pressure-spike [1,4-14] as the case may be. Note

231 that a minor disturbance to the supersonic flow creates shock waves in the downstream regions.

232 Therefore, bulging or tearing of the vessel will always be at the downstream region of the sonic 233 point (see Fig.2(a-o)). This physical situation could be forecast through reliable multi-phase,

234 multi-species in silico models [5-14] verified and validated at the Sanal flow choking condition.

235 Further discussion on in silico model is beyond the scope of this paper. Figure 3(a) shows the 236 idealized physical model of an artery with the divergent region and Fig.3(b) shows the 237 corresponding SANAL chart (the solution of Eq.5a) relating to a case of gas embolism with 238 carbon dioxide as the chief evolved gas. Figure 3(b) explicitly reveals that irrespective of the 239 percentage blockage of the artery, the critical BPR determines the risk of the biofluid/Sanal flow 240 choking leading to asymptomatic cardiovascular diseases. The SANAL chart also confirms that 241 a reduction in the blockage factor decreases the flow Mach number for satisfying the 242 conservation law of nature (i.e., continuity condition set by nature), which reduces the risk of 243 internal flow choking. It is crystal clear from the closed-form analytical model that for negating 244 the internal flow choking all subjects must retain BPR always lesser than the LCHI. Analytical 245 model presented herein verifies that the stents could reduce the risk of the heart attack (i.e., due 246 to the reduction in the flow Mach number) but it is not better than drug owing to the fact that 247 the biofluid/Sanal flow choking could happen with and without stent (see Fig.2(d-f)) at a critical 
248 BPR. The self-explanatory equations (see Eqs. 1-5), derived from the compressible flow theory $249[5,6]$, are highlighting various influencing parameters for prohibiting the biofluid/Sanal flow 250 choking in the artery. Note that the ejection fraction is reflected in Eq.2b in terms of 251 biofluid/blood flow rate (BFR). It is apparent from the closed-form-analytical model (see Eq.2b) 252 that the ejection fraction is not the lone factor for declaring the risk of AHF. It is coupled with 253 the local cross-sectional area of the vessel, local biofluid/blood velocity, local Reynolds number, $254 \mathrm{BHCR}_{\text {lowest }}$ and the local static pressure (i.e., diastolic blood pressure (DBP)). The local 255 Reynolds number $\left(\operatorname{Re}=\frac{\rho v D_{H}}{\mu}\right.$ can be estimated from the local hydraulic diameter of the vessel $256\left(\mathrm{D}_{\mathrm{H}}\right)$, the mean velocity of the biofluid/blood fluid $(v)$, the density of the biofluid/blood $(\rho)$, and 257 the dynamic viscosity of the biofluid/blood $(\mu)$. The Reynolds number $(R e)$ helps predict flow 258 patterns in different fluid flow situations. At low Reynolds number, flow tends to be dominated 259 by laminar (sheet-like) flow, while at high Reynolds number, flow tends to be turbulent. The 260 high turbulence level increases the boundary layer displacement thickness (i.e., the boundary 261 layer blockage factor) causing an early Sanal flow choking.

262 In high risk subjects (BPR close to LCHI and/or Mach number close one) a slight 263 oscillation in the BPR predisposes to the transient choking and the unchoking events heading to 264 arrhythmia. It is appropriate to mention here that, generally heart valve problems involve aortic 265 and mitral valves. It is possibly because of its geometric shape similar to CD nozzle flow 266 passage. Further deliberations of valve problems, aneurysm and arrhythmia is beyond the scope 267 of this article. Note that the biofluid/Sanal flow choking could create unusual transient pressure268 overshoot in vessels with divergent/bifurcation regions [4-14], which increases memory effects 269 (stroke history) leading to artery tear in the subsequent stroke. The magnitude of the pressure- 
270 overshoot depends on the strength of the shock. It is decided by the flow Mach number and the

271 heat capacity ratio of the fluid.

\section{In vitro Methodology}

273 We have estimated the heat capacity ratio (HCR) of blood, obtained from the healthy human 274 being and one male Guinea pig living in the southern part of the Indian union, using the 275 Differential Scanning Calorimeter (DSC) - Perkin Elmer DSC 8000. Blood samples of healthy 276 subjects are obtained from an approved blood bank in India, viz., Bangalore Blood Bank \& 277 Diagnostic Laboratory (A Unit of Indian Trust For Social Action, License Number: 278 KTK/28C/1/94). Samples are collected from healthy subjects after the informed consent. And 279 blood sample of healthy Guinea pig is directly obtained from the animal living in the 280 Government of India approved Animal House Facility associated with the Faculty of Pharmacy M.S.Ramaiah University of Applied Science, Bangalore - 560054, INDIA (Registration number: 220/PO/ReBi/2000/CPCSEA). Please note that none of the Authors handled the animal for withdrawing blood from a four-week old healthy male Guinea pig. The guinea pig blood sample was drawn by an authorized person and provided to us by the officials of Animal House Facility upon our request. All the experimental methods reported herein are in accordance with relevant guidelines and regulations. Please note that the ethical committee approval is not required by 287 the national legislation of Indian union for conducting the blood sample test of healthy human 288 being and animals reported herein, which are applicable to all the authors and their affiliated 289 institutions in India. We confirm that all experimental protocols were approved by the National 290 Centre for Combustion Research and Development (NCCRD) of the Indian Institute of Science 291 (IISc) for the blood sample tests of healthy human being and animals. Also note that for 292 randomized studies the blood bank who supplied blood samples of healthy subjects obtained the 
293 written and informed consent from all the healthy human being prior to the test conducted at

294 NCCRD/IISc, India. The in vitro methodology is available at https://osf.io/p7kmg [10].

295 The Guinness Book of World Records (2014) reveals that a person with a reported heatstroke

296 having a body temperature of $115^{\circ} \mathrm{F}\left(46.1{ }^{\circ} \mathrm{C}\right)$ is the highest-reported body temperature, who

297 survived after medical care. Therefore, in this pilot work we have carried out detailed in vitro

298 studies beyond the highest-reported body temperature for different research objectives. Table-

2991 and Table-2 show the measured BPR, BHCR (estimated using in vitro method) and UCHI

300 (analytically predicted using Eq.4) of healthy subjects of age 23-56.

301

302

Table-1 Prediction of the $U C H I$ from the heat capacity ratio of fresh blood samples of healthy human being of age 23-56.

\begin{tabular}{|c|c|c|c|c|c|}
\hline Batch No. & $\begin{array}{c}\text { Blood } \\
\text { Group }\end{array}$ & $\begin{array}{c}\text { SBP/ } \\
\text { DBP }\end{array}$ & BPR & BHCR & $\begin{array}{c}\text { UCHI } \\
@ ~ 37.5^{\circ} \text { C }\end{array}$ \\
\hline 3073 & O+ & $150 / 90$ & 1.666 & 3.5 & 3.11 \\
\hline 3074 & A+ & $120 / 70$ & 1.714 & 2.76 & 2.691 \\
\hline 3078 & B- & $150 / 90$ & 1.666 & 2.7292 & 2.709 \\
\hline 3080 & O+ & $150 / 90$ & 1.666 & 2.9935 & 2.824 \\
\hline 3082 & A+ & $140 / 96$ & 1.458 & 2.6759 & 2.64 \\
\hline
\end{tabular}

304

305

306

Table 2. Upper Critical Hemorrhage Index (UCHI) of Healthy Subjects [10]

\begin{tabular}{|c|c|c|c|c|c|c|}
\hline $\begin{array}{l}\text { Specimen } \\
\text { Reference }\end{array}$ & \multirow{2}{*}{$\begin{array}{c}\text { SBP/DBP } \\
m m ~ H g\end{array}$} & \multirow{2}{*}{$\mathrm{BPR}$} & \multicolumn{2}{|c|}{$\begin{array}{c}\text { Heat Capacity Ratio } \\
(\mathrm{HCR} \text { ) of Healthy } \\
\text { Subjects }\end{array}$} & \multicolumn{2}{|c|}{$\begin{array}{c}\text { Upper Critical } \\
\text { Hemorrhage Index } \\
\text { (UCHI) }\end{array}$} \\
\cline { 4 - 7 } & & & $37.5^{\circ} \mathrm{C}$ & $40^{\circ} \mathrm{C}$ & $37.5^{\circ} \mathrm{C}$ & $40^{\circ} \mathrm{C}$ \\
\hline $\mathrm{HM} 35 \mathrm{~A}+$ & $110 / 76$ & 1.44 & 5.69 & 5.37 & 4.33 & 4.15 \\
\hline $\mathrm{HM} 23 \mathrm{~A}+$ & $130 / 60$ & 2.16 & 118.29 & 20.42 & 61.75 & 12.10 \\
\hline $\mathrm{HM} 48 \mathrm{~B}+$ & $110 / 80$ & 1.37 & 7.44 & 7.03 & 5.28 & 5.06 \\
\hline $\mathrm{HM} 37 \mathrm{O}+$ & $120 / 60$ & 2 & 18.07 & 6.39 & 10.88 & 4.71 \\
\hline
\end{tabular}




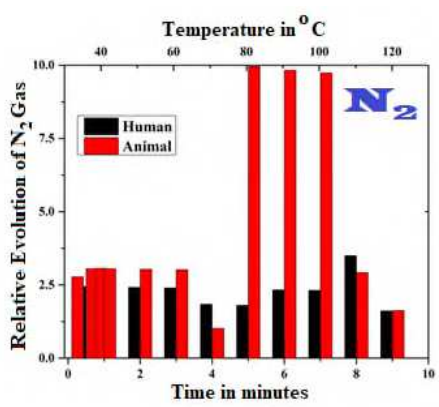

(a)

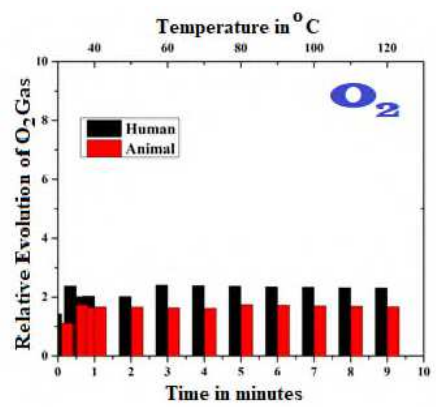

(b)

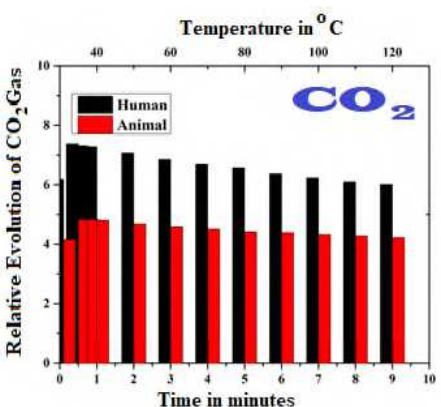

(c)

Fig. 4(a-c) The mass spectrum of $\mathrm{N}_{2}, \mathrm{O}_{2}$, and $\mathrm{CO}_{2}$ evolved as a function of both time and temperature obtained from blood samples of healthy subjects.

Figure 4(a-c) shows the mass spectrum of $\mathrm{N}_{2}, \mathrm{O}_{2}$, and $\mathrm{CO}_{2}$ evolved as a function of both time and temperature obtained from blood sample tests of healthy subjects (human being and Guinea pig). The mass spectrometer used in the present study is Perkin Elmer SQ8T, which uses the Electron Impact detector. During our comprehensive in vitro studies, we have noticed that the gases evolved from the fresh blood sample depends on the blood temperature, the heating rate, blood group, age and the blood pressure value. It is evident from Fig.4(c) that $\mathrm{CO}_{2}$ is the dominant gas for human being whereas nitrogen gas is dominant (Fig.4(a)) in the blood sample of Guinea pig.

The estimated LCHI of all healthy human being is found 1.82, which is based on the evolved dominant carbon dioxide gas $(\mathrm{BHCR}=1.289)$. In the case of Guinea pig, the LCHI is estimated as 1.89 , which is based on the dominant nitrogen gas $(\mathrm{BHCR}=1.4)$. We found that there are variations in the heat capacity of blood samples collected in three different Vacutainers of same healthy subjects. The anticoagulant reduces the BHCR and susceptible to an early biofluid choking in blood vessels, including vasa vasorum, causing high cardiovascular risk. The most popular consequence of medication with anticoagulant drug is bleeding. The clinical reports of 
327 various investigators [2, 3, 24, 27-30, 43] are corroborating the authenticity of our analytical 328 models and in vitro data reported herein.

329 It is crystal clear from Fig.4 that the possibilities of the Sanal flow choking in the animal

330 (Guinea pig) is lower than in the human being at the same temperature level as the heat capacity

331 ratio (HCR) of the main gas generated in the animal is found constantly higher than the human

332 being. The mass spectrum of $\mathrm{N}_{2}$ is observed greater in Guinea pig whereas in the healthy human

333 being $\mathrm{CO}_{2}$ is observed greater. The $\mathrm{HCR}$ of $\mathrm{N}_{2}$ is 1.4 and that of $\mathrm{CO}_{2}$ is 1.289 . It corroborates

334 that at the same thermal loading condition, the artery of Guinea pig gets choked only at a BPR 335 of 1.8929 and the artery of the healthy human being gets an early choking at a BPR of 1.8257 .

336 Therefore, we concluded that the thermal tolerance level of the healthy Guinea pig is higher and 337 the cardiovascular risk is lower than the human being under identical conditions. Therefore, 338 increasing the thermal tolerance level of the human being is important for reducing the risk of 339 AHF due to the Covid-19 or otherwise. More specifically, if BHCR is relatively high the 340 evaporation temperature of blood will also become high and as a result the risk of gas embolism 341 followed by internal flow choking can be reduced.

\section{In Silico Methodology}

343 In an effort to demonstrate the proof of the concept of BLB factor persuaded Sanal flow 344 choking we have carried out in silico studies with low-subsonic inflow (i.e., creeping flow) 345 conditions (a case with gas embolism) using a validated flow solver [9]. We have demonstrated 346 the pressure overshoot at the downstream region of the simulated artery with a divergent region 347 (see Fig.5) causing asymptomatic hemorrhage and/or acute heart attack as the case may be. 348 The preliminary single phase in silico results (see Fig.5) show the Sanal flow choking and the 349 shock-wave generation at the creeping flow heading to the transient pressure-overshoots 
350 (stroke) in the downstream region of an artery with divergent port. It substantiates that the 351 transient episode of biofluid/Sanal-flow-choking is a paradigm shift in the diagnostic sciences 352 of acute-heart failure.

353

354

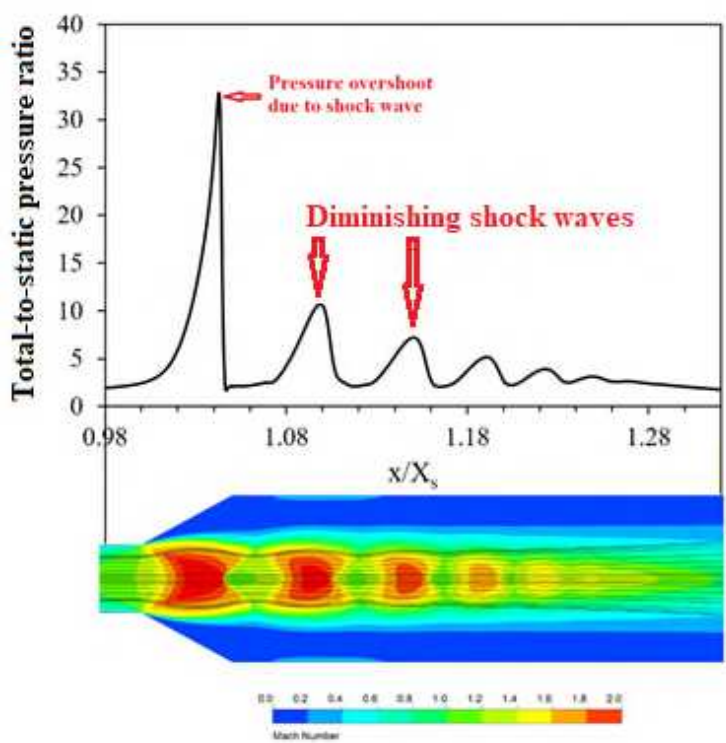

Fig. 5 In silico results show the Sanal-flow-choking and shock-wave generation at the subsonic inflow condition (creeping flow) leading to the transient pressure overshoots (causing memory effect / stroke) in the downstream region of an artery (where tissue death (infarction) occurs) with divergent port as a result of the CD nozzle flow effect (a case with gas embolism) [7, 8].

It is evident from Fig.5 that when static pressure decreases (i.e., DBP in biological systems) the chances of attaining the Sanal flow choking condition is very high. Note that flow choking is uniquely is regulated by BHCR. Therefore, total-to-static pressure ratio (i.e., systolic-todiastolic blood pressure ratio (BPR)) and BHCR are important determinants in biological systems for identifying the asymptomatic cardiovascular risk. The diminishing shock waves followed by the transient pressure-overshoots create memory effects in multiple locations of the 
365 artery during the entire lifespan of all subjects having oscillating BPR creating choking and

366 unchoking physical situations.

\section{Statistical analysis}

368 All in vitro studies were carried out independently at least six times for repeatability and also

369 for establishing that the data generated are in agreement with the true value in each independent 370 experiment. In silico studies are carried out after the code validation.

\section{Outcomes}

372 In vitro study proved that the specific heat of blood at constant pressure $\left(\mathrm{C}_{\mathrm{p}}\right)$ is always higher 373 than the specific heat at constant volume $\left(\mathrm{C}_{v}\right)$. Therefore, the validity of the analytical models 374 (Eqs.1-4) derived from the compressible flow theory for predicting the risk of flow choking 375 leading to $\mathrm{AH}$ and $\mathrm{AHF}$ presented herein is established. During the Hyphenated techniques at 376 the atmospheric pressure we have detected $\mathrm{N}_{2}, \mathrm{O}_{2}, \mathrm{CO}_{2}$, Ar and one undefined composite gas $377(\mathrm{~m} / \mathrm{z}=28.5)$ in blood samples of healthy subjects at various intensity at the temperature range 378 of $37-40^{\circ} \mathrm{C}\left(98.6-104^{0} \mathrm{~F}\right)$ and above [10]. We observed that the gasification temperature of 379 healthy Guinea pig blood is higher than the healthy human being. The BHCR of healthy subjects 380 taken from the EDTA and Lithium Heparin tubes was found significantly lower (31-32\%) than 381 the fresh blood samples of the same healthy subjects tested within 5 minutes of collection. We 382 observed that $\mathrm{CO}_{2}$, the gas with the lowest HCR is relatively and consistently higher in the 383 healthy males than the healthy male Guinea pig of four weeks old (see Fig.4). Note that HCR 384 of $\mathrm{CO}_{2}$ is 1.289 , therefore a subject with gas embolism, with $\mathrm{CO}_{2}$ as the dominant gas, the 385 biofluid choking occurs (see Eq.1) at a BPR of 1.8257, which is the lower critical hemorrhage 386 index (LCHI). It reveals that patients who are taking blood-thinning medication must maintain 387 their BPR always less than 1.8257, as dictated by Eq.1, for reducing the risk of internal flow 
388 choking leading to asymptomatic vascular diseases [27, 28]. This condition is particularly 389 important for subjects with relatively low BHCR.

390 The closed-form analytical model, in vitro and in silico study results reported herein reveal 391 that for a healthy-life all human being/animals with the high BPR inevitably have high BHCR 392 for reducing the risk of AHF by prohibiting biofluid/Sanal-flow-choking heading to shock wave 393 generation and transient pressure-spike causing memory effect (stroke history). The preliminary 394 single phase in silico results (see Fig.5) show the phenomenon of Sanal-flow-choking and shock 395 wave generation at the subsonic inflow condition (creeping flow) leading to the transient 396 pressure-overshoots (stroke) in the downstream zone of an artery with a divergent port.

\section{Results}

398 We discovered that at a critical blood-pressure-ratio (BPR), the internal-flow-choking occurs 399 anywhere in the cardiovascular system (CVS) with the sudden expansion/divergence/ 400 bifurcation / stenosis / occlusion or vasospasm regions. The critical fact is that; the internal401 flow-choking is uniquely regulated by $B H C R$. Analytical findings reveal that the relatively high 402 and low blood viscosity are cardiovascular risk factors. Equation 2a discloses the conflicting 403 requirements for achieving the unchoked flow condition for reducing the risk of AH and AHF. 404 The fact is that while using over dose of blood thinners, blood viscosity reduces and Reynolds 405 number increases and flow becomes turbulent. It creates an early flow choking due to the 406 development of the higher boundary layer blockage factor. Note that the turbulent boundary 407 layer displacement thickness is higher than laminar boundary layer thickness. Herein we 408 established that the disproportionate blood-thinning treatment increases the risk of internal flow 409 choking due to the enhanced boundary-layer-displacement-thickness (boundary layer blockage 
410 (BLB) factor) due to an increase in flow turbulence in the CVS due to an increase in Reynolds

411 number $(R e)$ as a consequence of relatively low blood viscosity (BV).

\section{Discussion}

413 Through closed-form analytical models (see Eqs.1-4) we could correlate the BHCR, BPR, 414 blood viscosity, port geometry of the vessel and ejection fraction, along with other parameters 415 contributing for internal flow choking. The infallible closed-form analytical models with the 416 multitude variables shed light on finding solutions for decreasing the risk of AH and AHF due 417 to biofluid choking and/or Sanal flow choking. Note that internal flow choking leads to shock wave 418 generation in CVS (see Fig.2(a-o). Please note that the internal flow choking (biofluid choking 419 and/or Sanal flow choking) is a physical situation created by nature at a critical-pressure-ratio 420 (cpr) (please see the supplementary materials Movie S1, S2, \& S3). In biological systems, the $421 \mathrm{CPR}$ is the ratio of systolic-to-diastolic blood pressure $(\mathrm{BPR}=\mathrm{SBP} / \mathrm{DBP})$. It is uniquely 422 controlled by biofluid/blood-heat-capacity-ratio (BHCR). It is a well-known scientific fact that 423 the internal flow choking leads to supersonic flow development if the downstream region of the 424 vessel geometry is having divergence and/or bifurcation regions. It is important to note that a minor 425 disturbance to the supersonic flow leads to shock wave generation and transient pressure-overshoot 426 and memory effects (stroke history) causing asymptomatic cardiovascular episodes. This is 427 corroborated with the observation of Packer [17]. More specifically, shock wave is inherently a 428 transient phenomenon, which create transient pressure-overshoot causing acute-heart-failure 429 (AHF). It is important to note that multitudes of variables highlighted on the causes and effects 430 of $\mathrm{AH}$ and AHF, over the decades, are supported with our findings [1, 4-14]. Briefly, herein, we

431 could establish, based on the infallible closed-form analytical models supported with in vitro and in 432 silico results, that $\mathrm{AH}$ and $\mathrm{AHF}$ are transient-events due to flow choking and shock wave 
433 generation. Please note that the exact solution obtained from the closed-form analytical model

434 is not required any verification using in vitro data. Nevertheless, in vitro data generated from

435 the blood sample test of healthy subjects provided herein for corroborating our theoretical

436 discovery. In vitro data is also used for establishing the unchoked flow condition in healthy

437 subjects having relatively high $\mathrm{BPR}(>1.8257)$ and BHCR (see Table-2). The occurrence of

438 choked flow condition and shock wave generation causing hemorrhagic-stroke is demonstrated

439 using the clinical data of a Covid-19 patient [1]. Briefly, there is no need of large data base for

440 establishing the choking and unchoking conditions in cardiovascular system. More specifically,

441 the accurate measurement of BPR and the high fidelity in vitro estimation of BHCR of all

442 subjects are sufficient for the risk assessment of asymptomatic cardiovascular diseases due to

443 the phenomenon of internal flow choking.

444 We have discovered through closed-form analytical models that if we increase the thermal

445 tolerance level in terms of blood heat capacity ratio (BHCR) and/or decrease BPR, we can 446 control concurrently blood viscosity and turbulence and prohibit undesirable flow choking (see

447 Eq.1-4). This is a remarkable finding reported herein. At the threshold of the internal-flow448 choking condition, a minor oscillation in BPR for both hyper and hypo subjects is likely to 449 aggravate the risk of the asymptomatic cardiovascular disease. Our finding is corroborated with 450 the clinical report presented by Razavi et al. [43] (PMID: 32322398; PMCID: PMC7163302; 451 DOI: 10.1016/j.nmni.2020.100669) from Mazandaran University of Medical Science, 452 Iran. Note that if BHCR is relatively low (i.e., low thermal tolerance) blood will get vaporize 453 early in Covid-19 patients and others leading to gas embolism. At this condition if BPR is 454 increasing the chances of asymptomatic cardiovascular diseases will be high due to the 455 undesirable internal flow choking. Therefore, increasing the thermal tolerance level in terms of 
$456 \mathrm{BHCR}$ and/or decreasing the BPR is a possible remedy for negating the internal flow choking 457 causing $\mathrm{AH}$ and AHF. We discovered through analytical methodology that BHCR is a unique 458 parameter, which can control concurrently blood viscosity and turbulence. Briefly, high BHCR 459 reduces blood viscosity and turbulence.

460 Generally, we consider blood flow is laminar in the CVS. The fact is that blood flow 461 becomes turbulent while taking blood thinning drugs. It is well known that the blood thinning 462 medication reduces the whole blood viscosity (BV) and as a result Reynolds number $(R e)$ 463 increases and the laminar blood flow becomes turbulent. This physical situation results in the 464 shortfall of energy in the form of friction. It, obviously, increases the boundary layer blockage 465 (BLB) in the blood vessels. It generates heat and augment the internal energy causing a decrease 466 in BHCR, which is corroborated with in vitro results. Furthermore, turbulence augments the 467 perfusion pressure crucial to drive the blood flow.

468 The shear rate or shear rate history of the blood/biofluid can alter viscosity. Blood viscosity 469 could vary due to seasonal effects too [19-40]. Note that temperature of blood reduces during 470 the winter season causing a rise in viscosity of blood. During the summer season the inverse 471 effects happen [9]. All these lead to corroborate that the boundary layer blockage (BLB) factor 472 initiating the Sanal flow choking [4-7] could vary due to the variations in blood viscosity (BV) 473 as a result of the seasonal effects and/or due to the usage of the blood-thinning drugs [9]. Truly, 474 BLB persuaded flow choking (biofluid/Sanal flow choking) is more susceptible in the winter 475 season than the summer season because the viscosity of blood will be relatively high at a low 476 temperature. Therefore, the risk of flow choking would be high during the winter season than 
477 in the summer season. It leads to AH and AHF. These findings are corroborating with the 478 available literature data [6-14, 24].

479 Equation 1 reveals that the critical ratio of BPR for flow choking is an exclusive function 480 of BHCR. It is crystal clear from Eq.2a that stenosis could increase the risk of flow choking. 481 Note that in Eq.2a, stenosis is taken in terms of the hydraulic diameter of the blood vessel. Eq.2a 482 also tells us that stent fix for raising the hydraulic diameter of the vessel could reduce the risk 483 of flow choking. Closed-form analytical models presented herein reveal that stent implant is 484 certainly not a stable solution for lessening the risk of AHF, without having suitable control on 485 the other multitude parameters highlighted in the models (see Eqs.1, 2, 2a \& 2b).

486 Though all the prevailing percentage demarcations of the ejection fraction (EF) are 487 significant for the diagnosis, until the theoretical discovery of Sanal flow choking [1,4-14] these 488 outcomes were not aided by any closed-form analytical model for taking any reliable 489 conclusion. Therefore, we have reported herein the infallible closed-form analytical models for 490 establishing the internal flow choking with the multitude of variables (see Eqs.1-4). Equation $4912 \mathrm{~b}$ explains the desirable biofluid/blood flow rate (BFR) or ejection fraction for predicting the 492 risk of AHF. Equations 2a-b are showing the correlation of the ejection fraction in terms of local 493 BFR and the flow turbulence level in terms of Reynolds number of normal heart along with the 494 other governing factors viz., vessel geometry, thermodynamic and fluid dynamics properties of $495 \mathrm{blood} / \mathrm{biofluid}$. These factors contribute for flow choking and unchoking in CVS at various 496 seasons. It is evident from Eqs.2a-b that the risk factor changes on the coupled effects of the 497 ejection fraction, the local vessel cross-sectional area (VCA), BHCR, DBP and local blood 498 viscosity. Mathematical models presented herein reveal that, at a constant VCA and DBP a 499 reduction in BHCR and a rise in Reynolds number together or separately, increases the 
500 likelihoods of internal flow choking. Seemingly (see Eq.2a) a rise in kinematic viscosity 501 increases the risks of flow choking. On the contrary, a rise in kinematic viscosity decreases the 502 risks of flow choking by reducing the Reynolds number. This infallible analytical finding reveals 503 that there is a safe range of blood viscosity for negating the flow choking of each and every 504 subject. Note that flow choking depends up on the joint effects of the other governing factors 505 (see Eqs.1, 2, 2a \& 2b). Therefore, in the absence of BHCR data (still unknown to medical 506 science), the dose of blood-thinning drugs must be recommended subjected to the condition set 507 in Eqs.2a \& 2b based on the clinical data (multitude of variables highlighted in Eq.1-5) of 508 individual subject.

509 Note that using over-dose of drug to decrease the blood viscosity only makes the turbulence 510 worse and escalates the possibilities of cavitation and flow choking because as stated earlier 511 turbulent flow creates higher BLB factor than laminar flow. The BLB in the blood vessel 512 generates heat and augment the internal energy causing a decrease in BHCR, which is vulnerable 513 to an early flow choking in the cardiovascular system (CVS). On these rationale, we established 514 herein that the uneven blood-thinning medications increase the possibilities of internal flow 515 choking causing AHF, which is supporting with the established laboratory index, International 516 normalized ratio (INA). Briefly, an overdose of blood-thinning drug enhances the Reynolds 517 number causing to the large turbulence level in the vessel and as a result the laminar flow could 518 be intruded and converts to turbulent flow causing an early internal flow choking (biofluid 519 and/or Sanal flow choking). Flow choking causes a transient sharp pressure-spike as a result of 520 the occurrence of shock waves, at the creeping inflow condition without any iota of symptoms 521 of the plaque, in an artery with sudden expansion / divergence / bifurcation / vasospasm (see 522 Fig.2(a-o) as the Central Illustration). Admittedly, internal flow choking can develop in 
523 nanoscale vessels [1] and also in the coronary artery with and without stent (Percutaneous

524 Coronary Intervention). When the pressure of the nanoscale fluid increases, the average mean 525 free path decreases and thus, the Knudsen number reduces leading to a no-slip boundary 526 condition with compressible viscous (CV) flow effect [1]. The Sanal-flow-choking is a CV flow 527 effect. It is established as the flow choking caused by the BLB factor at the creeping inflow 528 condition at a critical pressure ratio. Briefly, the discovery of internal flow choking in CVS is a 529 scientific breakthrough and a paradigm shift in the diagnostic science of asymptomatic vascular 530 diseases. Internal flow choking (biofluid/Sanal flow choking) leads to the shock-wave 531 generation followed by pressure overshoot causing tearing of the blood vessels. The tearing 532 depends on the memory effects (stroke history) and the thermo-viscoelastic properties of the 533 vessel. This fundamental research paper, started from the chemical rocket science [1,6,9], aims 534 to discover the basic cause(s) of bleeding during the administration of blood-thinning drugs. It 535 also aims to recommend possible conditions for reducing the risk of internal flow choking 536 causing $\mathrm{AH}$ and AHF.

537 Note that large swings in BPR create periodic choking and unchoking phenomena causing 538 atrial fibrillation (AFib) or an irregular heartbeat (arrhythmia) in both hyper and hypo subjects. 539 In light of the Covid-19 pandemic, the thermal tolerance level of blood needs to be examined in 540 terms of variations in the BHCR for the risk assessment of the ischaemic heart disease. The 541 European Society of Cardiology (ESC) reported (2020) that subjects with cardiovascular risk 542 (CVR) factors and proven cardiovascular disease (CVD) denote an open population when 543 suffering from the Covid-19. ESC also added that subjects with cardiac injury in the assessment 544 of Covid-19 have a greater risk of illness and demise (www.escardio.org). This article sheds 545 light for discovering new avenues in biological science for devising new blood-thinning drugs 
546 for increasing BHCR and/or decreasing BPR. Note that companion drugs along with the

547 traditional blood thinners for reducing the risk of flow choking causing AH and AHF [1, 6-14,

$54842]$ is also envisaged. We concluded that the cardiovascular treatment should be targeted based

549 on blood pressure ratio (BPR), instead of blood pressure levels alone, along with the BHCR

550 optimization, in chronic heart failure patients [40].

551 The intermittent internal flow choking, due to large fluctuations in BPR, leading to transient

552 pressure-overshoots created throughout the life-span in the CVS create the vessel walls more

553 stiff due to memory effects (stroke history). Such intermittent flow choking in any viscoelastic

554 vessels having stenosis/divergence/bifurcation regions are prone to rupture, possibly the

555 downstream region from sonic point, in the subsequent choking and the shock wave

556 development. Briefly, we have reported conclusively herein (see Eq.1) that high BPR and low

557 BHCR are risk factors for the flow choking causing transient episodes in CVS. An elevated

558 systolic BP (SBP) and lower diastolic BP (DBP) reflects arterial aging [41]. It happens due to

559 the repeated Sanal-flow-choking and "shock wave generation" causing memory effects ending

560 with so-called natural mortality due to the life-threatening-vessel tearing due to gradual

561 stiffness. The physical situation of Sanal-flow-choking is more dangerous in Covid-19 patients,

562 which could lead to stroke epidemic. In high risk subjects, (i.e., BPR is very close to the LCHI),

563 a slight oscillation in the BPR predisposes to the choking and the unchoking phenomena, which

564 could lead to arrhythmia and memory effect. Briefly, this study sheds light for exploring new

565 avenues in biological science for discovering the actual cause of "natural mortality" through

566 autopsy for devising new drugs for increasing the healthy lifespan of all subjects in the universe

567 (on earth, human space station and other planets). Discovering a companion medicine with the 
568 traditional blood-thinning drugs for reducing the risk of internal flow choking causing 569 asymptomatic cardiovascular diseases is also envisaged herein.

\section{Conclusions}

571 An over dose of blood-thinning drug will enhance the Reynolds number, which creates high

572 turbulence level causing an augmented boundary layer blockage factor leading to an early 573 undesirable biofluid/Sanal flow choking. We concluded through infallible closed-form 574 analytical models that relatively high blood viscosity and relatively low blood viscosity are 575 risk factors for the early flow choking. It leads to asymptomatic-hemorrhage (AH) and the acute576 heart-failure (AHF). These findings are correlating with the established index INA. Therefore, 577 the actual effect of viscosity on flow choking needs to be established for taking preventive 578 strategies for negating the risk of $\mathrm{AH}$ and $\mathrm{AHF}$. On this rationale, it is desirable, rather 579 necessary, perhaps inevitable to affirm a condition for preventing the undesirable flow choking 580 in the CVS, which we have presented herein in terms of blood viscosity, density, Reynolds 581 number, BHCR, BPR, DBP and stenosis (vessel geometry). We concluded that a single drug 582 capable to enhance the BHCR and suppress the turbulence level and/or a companion drug along 583 with the conventional blood-thinning drug is inevitable for meeting the conflicting requirements 584 (i.e., decrease viscosity and turbulence simultaneously) for prohibiting the flow choking of all 585 the subjects for reducing the risk of asymptomatic vascular diseases. In high risk subjects (BPR 586 close to the $L C H I$ ) a minor fluctuation in the blood pressure ratio (BPR) influences to the 587 choking and the unchoking phenomena, which could lead to arrhythmia. In a nutshell, we have 588 ascertained decisively that low BP ratio and the high-BHCR reduce the risk of flow choking as 589 dictated by Eq.1, which is an indisputable physical condition, without any ex vivo or in vivo 590 model support, for prohibiting asymptomatic stroke in any vessel [34-37]. We concluded that 
591 for a healthy-life all subjects with high-BP ratio (BPR) inevitably have high-BHCR for reducing 592 the risk of internal flow choking (biofluid/Sanal-flow-choking) triggering the AH and AHF due 593 to the shock wave generation and transient pressure overshoot $[1,6-14,44,45)$. Note that 594 magnitude of the pressure-overshoot depends on the strength of the shock wave (normal/oblique 595 shock wave) [46]. We corroborated herein that, the acute-heart-failure (AHF) is a transient 596 episode [17] due to the internal flow choking followed by shock wave generation and transient 597 pressure-overshoot.

598 Study limitations

$599 \quad$ Conducting in vivo studies in all subjects require ethical clearance.

\section{Translational Outlook}

601 Large randomized blood sample tests for BHCR estimation along with BPR measurement, 602 adequately in all seasons in all blood groups, across the globe are needed for discovering new 603 drugs capable to increase the BHCR and/or decrease the BPR in all seasons for reducing the 604 risk of internal flow choking in all subjects.

\section{Acknowledgments}

606 The first author thanks to SERB/DST, the Government of India, AIIMS, New Delhi and 607 NCCRD/IISc, Bangalore, India for the fruitful and coherent conclusion of this study.

608 Data availability statement

609 The data that support the findings of this study are available from the corresponding author upon 610 reasonable request.

\section{Code availability statement}

612 The mathematical algorithm used for generating analytical results are available with the author

613 VS. The code used for generating in vitro results are available with the authors RSB and CO. 
614 The code used for generating the in silico results is available with the authors NC and AS. The

615 raw data required to reproduce the results are available with the corresponding author and could

616 be shared upon reasonable request.

617

618

619

620

621

622

623

624

625

626

627

628

629

630

631

632

633

634

635

636

\section{References}

1. SanalKumar V.R et al., Discovery of Nanoscale Sanal Flow Choking in Cardiovascular System - Exact Prediction of the 3D Boundary-Layer-Blockage Factor in Nanotubes, Nature Scientific Reports, 29 July 2021, http://www.nature.com/articles/s41598-021$\underline{94450-8}$

2. Fifi, J. T., \& Mocco, J. COVID-19 related stroke in young individuals. The Lancet Neurology. 19(9), 713-715 92020). doi:10.1016/s1474-4422(20)30272-6

3. Ellul M A. et al. (2020). Neurological associations of COVID-19. The Lancet Neurology. 19: 767-783 (2020), doi:10.1016/s1474-4422(20)30221-0

4. SanalKumar, V. R., Vigneshwaran, S., Nichith, C., \& Sulthan Ariff Rahman, M. Discovery of SANAL flow choking phenomenon, Patent No. IN201841049355, India, Date of online publication: January 4, 2019.

5. SanalKumar, V. R. et al. A closed-form analytical model for predicting 3D boundary layer displacement thickness for the validation of viscous flow solvers," AIP Advances, 8, 025315 (2018), pp.1-22, doi: 10.1063/1.5020333.

6. SanalKumar, V. R. et al. Sanal Flow Choking: A Paradigm Shift in Computational Fluid Dynamics Code Verification and Diagnosing Detonation and Hemorrhage in RealWorld Fluid-Flow Systems, Glob Chall. 2020 May 27;4(9):2000012., Wiley Publication (2020), doi: $10.1002 / \mathrm{gch} 2.202000012$. 
637 7. SanalKumar, V. R., et al. Lopsided blood-thinning drug increases the risk of internal 638 flow choking and shock wave generation causing asymptomatic stroke, Stroke. 2021;52:AP804, American Stroke

Association

(2021), https://doi.org/10.1161/str.52.suppl_1.P804

641

8. SanalKumar V. R. et al. Lopsided blood-thinning drug increases the risk of internal flow choking leading to shock wave generation causing asymptomatic cardiovascular disease, Glob Chall. 2021 Jan 29;5(3):2000076, Wiley Publication (2021), doi: $10.1002 / \mathrm{gch} 2.202000076$.

9. SanalKumar, V. R., et al. Boundary layer blockage, venturi effect and cavitation causing aerodynamic choking and shock waves in human artery leading to hemorrhage and massive heart attack - A new perspective. AIAA AVIATION Forum, June 25-29, 2018, AIAA 2018-3962, 2018 Applied Aerodynamics Conference. doi:10.2514/6.2018-3962 10. SanalKumar, V. R., et al. Nanoscale flow choking and spaceflight effects on cardiovascular risk of astronauts - A new perspective, AIAA Scitech 2021 Forum, 1115 \& 19-21 January 2021, AIAA 2021-0357, https://doi.org/10.2514/6.2021-0357

11. SanalKumar, V. R., et al. Internal flow choking in cardiovascular system: A radical theory in the risk assessment of asymptomatic cardiovascular diseases, Book Chapter, IntechOpen, DOI: http://dx.doi.org/10.5772/intechopen.96987

12. SanalKumar, V. R., et al. COVID 19 pandemic: High BPR and low BHCR are risk factors of asymptomatic cardiovascular diseases, Virology \& Mycology Open Access, 2021, Vol.10 Iss.3 No:204 (2021), ISSN: 2161-0517 
13. SanalKumar, V. R., et al. Sanal flow choking leads to aneurysm, hemorrhagic stroke and other neurological disorders in earth and human spaceflight - New perspective, Mini Review, Journal of Neurological Disorders, ID \#jnd-21-28473, March 2021 (Accepted).

14. Sanal Kumar V.R, Bharath RS, Nichith Chandrasekaran, et al. High Heat Capacity of Blood Reduces Risk on Myocardial Infarction, World Congress On Cardiac Sciences, Bangalore, India http://cardiacsciencesconference.com/ (BioGenesis, The Journal of Biology and Medicine, Vol.1, November 2018, pp.41-45).

15. WHO

Mortality

Database. Geneva: World

Health Organization; 2018, http://www.who.int/healthinfo/mortality_data/en/, https://openwho.org/courses/pandemic-epidemic-diseases

16. Alexandra N. Nowbar, Mauro Gitto, James P. Howard, Darrel P. Francis, Rasha AlLamee, Mortality From Ischemic Heart Disease, Circulation: Cardiovascular Quality and Outcome, Vol.12, No.6, https://doi.org/10.1161/CIRCOUTCOMES.118.005375

17. Packer, M. (2018). Acute Heart Failure Is an Event Rather Than a Disease. JACC: Heart Failure, 6(1), 73-75. doi:10.1016/j.jchf.2017.05.008

18. Mebazaa, A. (2018). Acute Heart Failure Deserves a Log-Scale Boost in Research Support - Call for Multidisciplinary and Universal Actions. JACC: Heart Failure, 6(1), 76-79. doi:10.1016/j.jchf.2017.09.012

19. Craig T. January, L. Samuel Wann, Hugh Calkins et al., 2019 AHA/ACC/HRS Focused Update of the 2014 AHA/ACC/HRS Guideline for the Management of Patients With Atrial Fibrillation. Circulation, 2019; DOI: 10.1161/CIR.0000000000000665 
679 20. Shikdar, S, Bhattacharya, PT. International normalized ratio 680 (INR). StatPearls. https://www.ncbi.nlm.nih.gov/books/NBK507707/. Updated October 27, 2018. Accessed October 2019.

21. Ezekiel Uba Nwose, Nathan Cann, and Eugene Butkowski, N Am J Med Sci. 2010 Jul; 2(7): 301-305, doi: 10.4297/najms.2010.2301

22. M. Brust, C. Schaefer, R. Doerr, et al., "Rheology of Human Blood Plasma: Viscoelastic Versus Newtonian Behavior,” Phys. Rev. Lett. 2013, 110, 078305.

23. A.S. Varchanisa, Y. Dimakopoulosa, Christian Wagnerb, J. Tsamopoulosa, How viscoelastic is human blood plasma?, Soft Matter,2018, DOI: 10.1039/C8SM00061A.

24. Stewart S, Keates AK, Redfern A, and McMurray JV, "Seasonal variations in cardiovascular disease," Nat Rev Cardiol, $\quad 201714(11), \quad 654-$ 664. doi:10.1038/nrcardio.2017.76

25. Rongjia Tao, Reducing blood viscosity and suppressing turbulence with magnetic field

26. R. Tao and K. Huang, Phys. Rev. E 84, 011905 - Published 12 July 2011, https://doi.org/10.1103/PhysRevE.84.011905

27. Hans-Christoph Diener et al., N Engl J Med (2019); doi: 10.1056/NEJMoa1813959 
31. J.-M. Neuhaus, L. Sticher, F. Meins, Jr., T. Boller, Proc. Natl. Acad. Sci. 88, $10362-$ 10366 (1991).

32. E. van Sebille, M. Doblin, https://dx.doi.org/10.6084/m9.figshare.3178534.v2. Deposited 15 April 2016.

33. A. V. S. Hill, "HLA associations with malaria in Africa: Some implications for MHC evolution" in Molecular Evolution of the Major Histocompatibility Complex, J. Klein, D. Klein, Eds. (Springer, 1991), pp. 403-420.

34. Li, X., Fries, S., Li, R., et al., (2014).PNAS, doi:10.1073/pnas.1406997111

35. Wang, W., Shen, M., Fischer, C. et al.(2019). PNAS, doi:10.1073/pnas.1900152116

36. M. E. Lindsay, H. C. Dietz, Nature 473, 308-316 (2011).

37. Xu, D., Varshney, A., Ma, X. et al. (2020). PNAS, doi:10.1073/pnas.1913716117

38. Ma, Y., Choi, J., Hourlier-Fargette, A., Xue, Y. et al., (2018). Proceedings of the National Academy of Sciences, 201814392. doi:10.1073/pnas.1814392115

39. Lindsay, $\quad$ M. $\quad$ E., $\quad \& \quad$ Dietz, $\quad$ H. $\quad$ C. (2011). Nature, 473(7347), 308316. doi:10.1038/nature10145

40. Jesse F. Veenis, Hans-Peter Brunner-La Rocca, Gerard C.M. Linssen, et al., Treatment Differences in Chronic Heart Failure Patients With Reduced Ejection Fraction According to Blood Pressure, Circulation: Heart FailureVol. 13, No. 5, May 2020, https://doi.org/10.1161/CIRCHEARTFAILURE.119.006667

41. Rajiv Agarwal, Blood Pressure Components and the Risk for End-Stage Renal Disease and Death in Chronic Kidney Disease, Clin J Am Soc Nephrol. 2009 Apr; 4(4): 830837, doi: 10.2215/CJN.06201208, PMCID: PMC2666439 
42. ESC Guidance for the Diagnosis and Management of CV Disease during the COVID19 Pandemic, https://www.escardio.org/Education/COVID-19-and-Cardiology/ESCCOVID-19-Guidance.

43. Sharifi-Razavi, A., Karimi, N., \& Rouhani, N. COVID 19 and Intra cerebral hemorrhage: Causative or Coincidental. New Microbes and New Infections, 35, 100669 (2020). doi:10.1016/j.nmni.2020.100669, PMCID: PMC7163302

44. V.R.Sanal Kumar et al., Sanal Flow Choking Leads to Hemorrhagic Stroke and Other Neurological Disorders in Earth and Human Spaceflight, American Heart Association, Presented at the Basic Cardiovascular Sciences Conference, 23-25 August 2021, Circulation Research, October 2021 (Accepted) https://e.ctt.bz/e.asp?e=96DA276B-3868-4D8C-9064-B6DE996DDCA1

45. V.R.Sanal Kumar et al., Deflagration to Detonation Transition in Chemical Rockets with Sudden Expansion / Divergence Regions, 2020 AIAA Propulsion and Energy Forum, AIAA 2020-3520, https://arc.aiaa.org/doi/10.2514/6.2020-3520

46. Anderson, J. Jr., Modern Compressible Flow, with Historical Perspective, Fourth Edition, McGraw-Hill Publishing Company, 2007

\section{Author Contributions}

VRSK: Conceptualization, analytical modeling, manuscript drafting; SKC: In vitro conceptualization, manuscript editing; PKR: In vitro conceptualization, manuscript editing; RSB: In vitro and data generation; NC: In vitro, in silico and project support, VS: Modeling and simulation support, AS: In silico simulation support, CO: Resources 


\section{Supplementary Files}

This is a list of supplementary files associated with this preprint. Click to download.

- APPENDIX30August2021.pdf

- MovieS1DemonstratingBoundarylayerblockageinducedSanalFlowChokingInsilicoresult.avi

- MovieS2FlowchokingandShockwavegenerationinaViscoelasticTubewithVasospasmInvitroresult.mp4

- MovieS3flowchokingwithairasfluid.mp4 